\title{
Raciocínio Lógico Atrelado a um Projeto de Robótica Educacional: Desempenhos de Alunos do $7^{\circ}$ Ano do Ensino Fundamental
}

\author{
Logical Reasoning Linked to an Educational Robotics Project: 7th Year Students' \\ Performances of Elementary School
}

\author{
Ana Beatriz de Oliveira*a, Ana Caroline Frigéri Barboza ${ }^{a}$; Érica Gambarotto Jardim Bergamimª Fabíola Martins da \\ Silvab; Glaucio Pedro de Alcantara ${ }^{\mathrm{a}}$; Juliana Ferreira da Silva ${ }^{\mathrm{a}}$
}

\begin{abstract}
${ }^{a}$ Universidade Estadual de Maringá, Programa de Pós-Graduação Stricto Sensu em Educação para a Ciência e a Matemática. PR, Brasil.
${ }^{\text {b}}$ Rede Estadual de Ensino do Estado do Paraná. PR, Brasil.

*E-mail: anaabiaah@gmail.com
\end{abstract}

\begin{abstract}
Resumo
Estudos atrelados aos processos de aprendizagem de Matemática evidenciam dificuldades recorrentes que os alunos manifestam nessa disciplina. $\mathrm{Na}$ busca por alternativas pedagógicas para contornar essas dificuldades, uma possibilidade é o uso da robótica educacional. Nesse sentido, este escrito tem como objetivo verificar desempenhos ocorridos nos processos de resolução de questões envolvendo raciocínio lógico, com a participação de alunos do $7^{\circ}$ ano do Ensino Fundamental, no que diz respeito às fases iniciais e finais de um projeto de robótica. Evidenciase que ambas as fases correspondem a dois momentos de aplicação dessas questões, no início do projeto e ao término, respectivamente, em que a intenção foi verificar se houve desempenhos de uma fase para a outra, e quais seriam esses. Para tanto, neste trabalho foi utilizada a abordagem qualitativa, que teve como instrumento de constituição de dados os registros dos participantes ao responderem questões que envolvem raciocínio lógico. O processo de análise foi respaldado por uma interpretação com base nos registros dos participantes, os quais desencadearam quatro eixos classificatórios relacionados ao desempenho dos alunos; ademais, no que se refere a registros representativos de cada desempenho, foram feitas algumas ponderações em relação ao referencial teórico levado em consideração. Como resultados, conclui-se que as atividades desenvolvidas ao longo do projeto propiciaram o desenvolvimento do raciocínio lógico, por parte dos alunos, e isso contribui para possíveis mobilizações de conhecimentos matemáticos dos educandos, que apresentavam dificuldades na disciplina de Matemática.
\end{abstract}

Palavras-chave: Lógica. Matemática. Eixos Classificatórios.

\begin{abstract}
Studies associated to the Mathematics learning processes show recurrent difficulties that students manifest in this discipline. In the search for pedagogical alternatives to overcome these difficulties, one possibility is the use of educational robotics. In this sense, this paper aims to verify the performances that took place in the process of resolving issues involving logical reasoning, with the 7th year students' participation of Elementary School, concerning the initial and final phases of a robotics project. It is evident that both phases correspond to two moments of application of these questions, at the beginning of the project and at the end, respectively, whereby the intention was to verify whether there were performances from one phase to the next and what they would be. For this purpose, in this work the qualitative approach was used, which had as a tool for constituting data the participants' records when answering questions that involved logical reasoning. The data analysis process was supported by an interpretation based on the participants' records, which triggered 4 classificatory axes related to the students 'performance; in addition, regarding the representative records of each performance, some considerations were made concerning the theoretical framework taken into consideration. As results, it is concluded that the activities developed during the project provided the logical reasoning development by the students and this contributes to possible mobilizations of students' mathematical knowledge who presented difficulties in the discipline of Mathematics.
\end{abstract}

Keywords: Logic. Mathematics. Classificatory Axes.

\section{Introdução}

Corriqueiramente, os estudantes da disciplina de Matemática apresentam dificuldades no processo de aprendizagem dessa disciplina (BRASIL, 1997). Dessa forma, tem-se buscado possibilidades de tornar o ensino da Matemática mais interessante para os alunos (D'AMBROSIO, 1989), de maneira a mitigar algumas dificuldades. Uma das estratégias adotadas para minimizar essas dificuldades se constitui do uso de recursos tecnológicos (VAN DE WALLE, 2009), tais como: calculadora, softwares de geometria dinâmica, robótica educacional, dentre outros.

Diante desse contexto, enfatiza-se que este estudo se apropria de um dos recursos tecnológicos elencados anteriormente, a saber, a robótica educacional. Tal estudo está atrelado a dois momentos específicos do desenvolvimento do projeto de extensão da Universidade Estadual de Maringá - UEM, intitulado "Projeto Lapidando Joias: um resgate ao futuro" ", que se refere à aplicação de questões em duas fases - sendo a primeira, Fase 1, no início do projeto; e a segunda, Fase 2, ao final do projeto. 
Entre as duas fases foram realizadas atividades diversificadas envolvendo a robótica, com o intuito de se aproximar do objetivo do projeto, que é possibilitar a elevação do desempenho escolar, em especial, na disciplina de Matemática, de alunos (carentes e com dificuldade de aprendizado) oriundos de escolas públicas com baixo IDEB através de um projeto que envolve o desenvolvimento do raciocínio lógico, a partir da utilização de kits educacionais de Robótica da empresa LEGO, bem como kits de Arduino ${ }^{2}$. No entanto, destaca-se que neste texto a robótica aparece apenas a título de indicação do contexto em que as atividades foram aplicadas.

No período de desenvolvimento deste trabalho, o projeto abrangia três escolas estaduais e uma escola municipal, localizadas na cidade de Maringá-PR, sendo o público-alvo alunos do $7^{\circ}$ ano e do $4^{\circ}$ e $5^{\circ}$ anos do Ensino Fundamental, respectivamente. Destaca-se que, conforme o objetivo do projeto, foram selecionados dez participantes de cada escola, com base em relatos de professores de que eles apresentavam dificuldades, principalmente, na disciplina de Matemática. No contexto deste trabalho, o enfoque se destina para somente uma das escolas participantes, sendo esta uma escola estadual.

Ressalta-se que a realização de projetos, como o que foi citado anteriormente, contribui para o desenvolvimento do pensamento computacional, conforme orientações apresentadas na Base Nacional Comum Curricular - BNCC (2018), uma vez que o pensamento computacional está relacionado ao desenvolvimento de algumas habilidades, como compreensão, análise, modelagem, resolução e solução de problemas, entre outras, de modo rigoroso e organizado por meio da elaboração e implementação de algoritmos.

Em consonância a isso, Brackmann, Caetano e Silva (2019) afirmam que o pensamento computacional possibilita a produção de habilidades de pensamento lógico e de resolução de problemas. Atrelada às habilidades de pensamento lógico está a problemática de pesquisa deste trabalho, a saber: que desempenhos podem ser verificados, por meio da aplicação de questões envolvendo o raciocínio lógico, na participação de alunos do $7^{\circ}$ ano do Ensino Fundamental em um projeto de robótica?

Ao encontro dessa problemática se tem como objetivo geral verificar desempenhos ocorridos nos processos de resolução de questões envolvendo o raciocínio lógico, com a participação de alunos do $7^{\circ}$ ano do Ensino Fundamental, no que diz respeito às fases iniciais e finais de um projeto de robótica.

Oraciocínio lógico sempre esteve atrelado ao conhecimento matemático. Essa ideia é corroborada por Oliveira e Rocha (2011), ao afirmarem que por mais que o pensar lógico e a matemática tenham sido formulados de modos distintos e independentes, há uma sinergia entre esses. Essa inferência também pode ser verificada no trabalho de Silva e Luna (2019), bem como em documentos oficiais que orientam os componentes curriculares de Matemática, como exposto na Base Nacional Comum Curricular (BNCC), ao evidenciar que entre as competências específicas de Matemática para o Ensino Fundamental consta o desenvolvimento do raciocínio lógico, do interesse pela investigação e argumentação persuasiva, usando-se os conhecimentos matemáticos para a compreensão e atuação em situações cotidianas (BRASIL, 2018).

Nesse sentido, faz-se importante destacar que o entendimento de raciocínio lógico adotado neste trabalho está em consonância com o que é apresentado por Oliveira e Rocha (2011). Tais autoras entendem raciocínio lógico com base em alguns parâmetros, os quais são denominados por: abstração, compreensão, argumentação com base em critérios e em princípios logicamente validados, expressão de ideias de forma lógica e organizada, números e suas relações (OLIVEIRA; ROCHA, 2011).

O parâmetro da abstração está relacionado com as ações dos participantes para o entendimento de conceitos sem a dependência do concreto, ou seja, de algo palpável, mas por meio de processos mentais; a compreensão, por sua vez, está vinculada ao ato de entender, interpretar e filtrar informações precisas de acordo com as necessidades requeridas; já o parâmetro da argumentação se trata da validação do pensamento, com base na reflexão dos próprios raciocínios e testes desenvolvidos para as ações realizadas, com critérios e princípios lógicos; quanto à expressão de ideias, de forma lógica e organizada, essa se refere a apresentar os pensamentos de maneira argumentativa e estruturada (OLIVEIRA; ROCHA, 2011).

$\mathrm{O}$ parâmetro número e suas relações está associado às relações que envolvem ramos da Matemática, estes são aritmética, geometria, álgebra, etc. Aqui, compreende-se que tal parâmetro está de acordo com o desenvolvimento do raciocínio lógico matemático, uma vez que, segundo Pontes et al. (2017), este é um processo de reorganização do pensamento, orientado por diretrizes da lógica, possibilitando a resolução de problemas aritméticos, geométricos ou matriciais, a fim de cooperar com habilidades cognitivas e aptidões dos estudantes.

Nesse sentido, o raciocínio lógico em uma visão geral se constitui como um elemento de relevância para o processo de resolução de problemas matemáticos (SCOLARI; BERNARDI, CORDENONSI, 2007), tendo em vista que para resolver problemas matemáticos é necessária a organização do pensamento, a formulação e teste de hipóteses, a elaboração

$2 \mathrm{O}$ Arduino que é um processador de código aberto baseado em hardware e software, de fácil manuseio. Esse tem várias entradas, que podem ser analógicas ou digitais, o que permite uma grande versatilidade, pois podem ser utilizados como sensores de entrada, sensores ópticos, botões, mensagens digitais por bluetooth, entre outros. Atualmente, a sua utilização em franca expansão em instituições de ensino, com vistas a construir instrumentos científicos de baixo custo, para iniciação à robótica e até mesmo para experimentos de física (CROVADOR, 2020). 
e estruturação de estratégias, a interpretação e validação dos dados. Concorda-se com Scolari, Bernardi e Cordenonsi (2007) no fato de que é essencial a compreensão e o raciocínio dos alunos perante o que é proposto e realizado, de modo que, não apenas, memorizem e apliquem fórmulas.

Para além de habilidades relacionadas apenas à Matemática, o raciocínio lógico contribui para a formação em um todo do indivíduo, tendo em vista que exercitá-lo é primordial para estruturar o pensamento e discernir informações. Dessa forma, em síntese, evidencia-se a importância do trabalho com atividades que envolvem o raciocínio lógico, como também o raciocínio lógico matemático em particular, como estímulo para alunos que possuem algumas dificuldades no processo de aprendizagem, com o intuito de possibilitar o desenvolvimento de habilidades relacionadas à resolução de problemas matemáticos e situações cotidianas, visando formação integral do aluno.

\section{Material e Métodos}

A pesquisa possui uma abordagem de natureza qualitativa, uma vez que segundo Rosa (2013, p.42) tem como intuito "utilizar instrumentos que privilegiem a qualidade da observação, normalmente estudando em profundidade poucos casos do evento, tentando identificar o que esses poucos casos têm em comum". Faz-se essa consideração por privilegiar os registros escritos dos participantes, buscando analisar os desempenhos que emergiram desses registros e, a partir disso, o que tais registros possuem em comum. Neste escrito, destaca-se que o entendimento da palavra "desempenho", que está em consonância com o que é definido pelo dicionário Michaellis (2020) como "Modo de executar uma tarefa que terá, posteriormente, seu grau de eficiência submetido a análise e apreciação".

Dessa forma, conforme abordado na introdução, o contexto a partir do qual se insere este trabalho faz referência ao projeto de robótica intitulado: "Projeto Lapidando Joias: um resgate ao futuro". Em relação à constituição dos dados se tem que esta ocorreu por meio da aplicação de questões envolvendo o raciocínio lógico, em duas fases, a saber: a primeira - Fase 1 -, ao início do projeto, e a segunda - Fase 2 -, ao término do projeto.

Ressalta-se que ambas as fases continham as mesmas questões, pois o intuito era averiguar o desempenho dos alunos de uma fase para a outra, o que vai ao encontro do objetivo deste trabalho. Além disso, salienta-se que os alunos tiveram em torno de 2 horas para responder as questões, tanto na primeira quanto na segunda fase.

Para mais, destaca-se que foram aplicadas dezesseis questões, entre as quais foram selecionadas sete, em função do fato destas apresentarem os parâmetros elencados no referencial teórico - abstração, compreensão, argumentação com base em critérios e em princípios logicamente validados, expressão de ideias de forma lógica e organizada, números e suas relações. Tais questões são apresentadas no Quadro 1.

Quadro 1 - Questões de raciocínio lógico

\begin{tabular}{|c|c|}
\hline Enunciados das Questões & Parâmetros Identificados \\
\hline 3. Mova 2 palitos e dobre o valor do número abaixo: ${ }^{3}$ & $\begin{array}{l}\text { Abstração (obs.: materiais); } \\
\text { compreensão; números e suas } \\
\text { relações }\end{array}$ \\
\hline $\begin{array}{l}\text { 4. Observe a sequência e escreva o próximo elemento da série: }{ }^{4} \\
\text { a) } 1,3,5,7, \overline{ } \\
\text { b) } 2,7,12,17,22,27 \\
\text { c) } 0,1,4,9,16,25,36\end{array}$ & $\begin{array}{l}\text { Compreensão; números e suas } \\
\text { relações }\end{array}$ \\
\hline $\begin{array}{l}\text { 8. Ana, Beatriz, Célia e Dora apostaram uma corrida. Ana disse: Célia ganhou, Beatriz chegou em } \\
2^{\circ} \text { lugar; Beatriz disse: Célia chegou em } 2^{\circ} \text { lugar e Dora, em } 3^{\circ} \text {; } \\
\text { Célia disse: Dora foi a última; Ana, a } 2^{\mathrm{a}} \text {; Cada uma das meninas disse uma verdade e uma } \\
\text { mentira. Qual a colocação de cada menina? }\end{array}$ & $\begin{array}{l}\text { Compreensão; expressão } \\
\text { de ideias de forma lógica e } \\
\text { organizada }\end{array}$ \\
\hline $\begin{array}{l}\text { 9. Assinale se as afirmações levam a uma conclusão Verdadeira (V) ou Falsa (F): } \\
\text { a) Todos os franceses são europeus. Descartes era francês.Logo, Descartes era europeu. } \\
\text { Verdadeira ( ) ou Falsa ( ) } \\
\text { b)Alguns engenheiros são professores. Nenhum engenheiro não comete erros.Logo, nenhum } \\
\text { professor não comete erros. } \\
\text { Verdadeira ( ) ou Falsa ( ) } \\
\text { c)Nenhum agricultor é rico. Todos os ricos são saudáveis.Logo, nenhum agricultor é saudável. } \\
\text { Verdadeira ( ) ou Falsa ( })^{6}\end{array}$ & $\begin{array}{l}\text { Compreensão; expressão } \\
\text { de ideias de forma lógica e } \\
\text { organizada }\end{array}$ \\
\hline
\end{tabular}




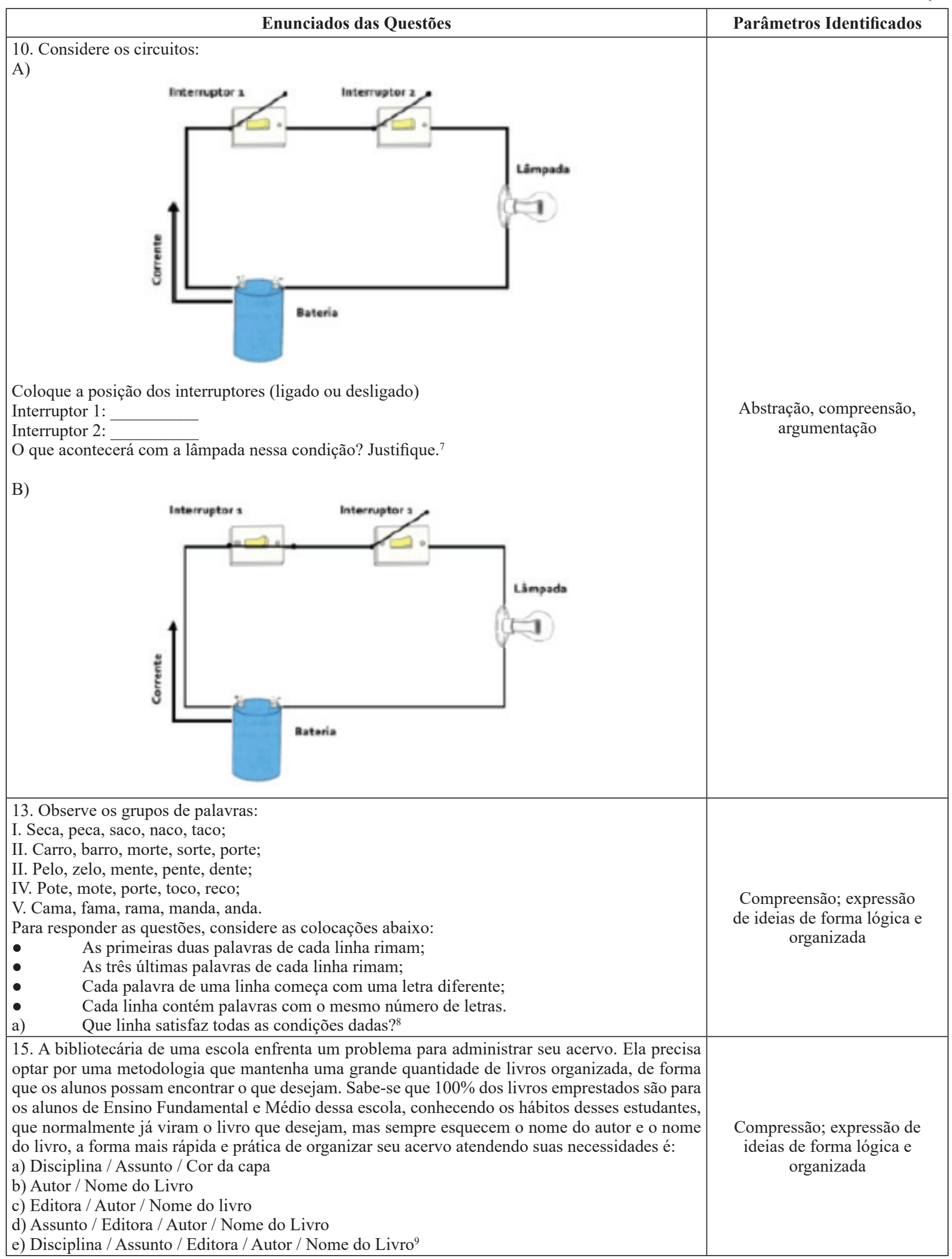

Fonte: os autores.

7 Retirado de Negreiros e Miranda (2015).

8 Retirado de Serates (1997).

9 Adaptado de Negreiros e Miranda (2015). 
No que se refere aos participantes envolvidos, destaca-se que todos os dez alunos responderam as questões aplicadas na Fase 1, enquanto na Fase 2 somente sete alunos as responderam. A ausência de três alunos na aplicação das questões da Fase 2 ocorreu pelo fato de problemas de saúde e por impossibilidade de caráter meteorológico; sendo assim, neste trabalho foram analisados somente os registros dos sete alunos que participaram das duas fases de aplicação.

O processo de análise dos dados foi respaldado por uma interpretação com base nos registros dos participantes, os quais desencadearam quatro eixos classificatórios relacionados aos desempenhos dos alunos. Primeiramente, para cada aluno foi realizada uma comparação das fases de aplicação, com base em cada questão, a fim de se verificar os desempenhos que seriam evidenciados. A partir disso se buscou agrupar os desempenhos comuns relativos aos participantes.

Para elucidar os casos verificados em cada eixo, foram apresentados quadros e, posteriormente, para exemplificar o que se entende por determinado desempenho, foram apresentados alguns registros dos participantes, um para cada eixo, tendo em vista que tais registros são representativos das ocorrências verificadas em cada classificação.

Ressalta-se que no registro selecionado para cada desempenho foi realizada uma discussão em relação ao referencial teórico deste trabalho, abrangendo os parâmetros de raciocínio lógico, conforme Oliveira e Rocha (2011). Na próxima seção serão elencados os desempenhos com suas respectivas análises.

\section{Resultados e Discussão}

\subsection{Desempenho 1: Não respondeu na Fase 1 e respondeu na Fase 2}

Neste eixo classificatório se enquadram os alunos que, na Fase 1, não responderam - deixaram em branco - uma ou mais questões e, na Fase 2, apresentaram registros nessas questões de forma correta.

Os casos que se encontram nesse eixo são correspondentes aos alunos A, C e F. Em relação ao aluno A, verifica-se a ocorrência desse desempenho nas questões 4b), 4c) e 9c). Por sua vez, o aluno C apresenta o desempenho somente na questão 3. Já o aluno F, evidencia o desempenho nas questões 3 e 4c). Podem-se observar tais informações no Quadro 2.

Quadro 2 - Casos em que ocorrem o desempenho 1

\begin{tabular}{|c|c|c|c|c|c|c|c|c|c|c|c|c|c|c|}
\hline Q & Q3 & & $\overline{\mathrm{Q} 4}$ & & Q8 & & Q9 & & & Q & & & Q13 & Q15 \\
\hline A & & $\mathrm{a}$ & b & c & & $\mathrm{a}$ & b & c & $\mathrm{a}$ & b & c & d & & \\
\hline Aluno $\mathrm{A}$ & & & $\mathrm{X}$ & $\mathrm{X}$ & & & & $\mathrm{X}$ & & & & & & \\
\hline Aluno $\mathrm{C}$ & $\mathrm{x}$ & & & & & & & & & & & & & \\
\hline Aluno $F$ & $\mathrm{X}$ & & & $\mathrm{X}$ & & & & & & & & & & \\
\hline
\end{tabular}

Fonte: os autores.
Para exemplificar o desempenho 1, foi escolhida a questão 4 , alternativas b e c, relativa ao aluno A, conforme se verifica no Quadro 3.

Quadro 3 - Registros do desempenho 1 do Aluno A com relação às fases 1 e 2 .

4. Observe a sequência e escreva o próximo elemento da série: a) $1,3,5,7$,

b) $2,7,12,17,22,27$,

c) $0,1,4,9,16,25,36$,

bustas (4) $9^{\text {Fase } 1}$

Fase 2
4) b) $2,9,12,14,22,27,32$
4) c) $0,1,4,9,16,25,36,49$

Fonte: os autores.

Nesse sentido, evidencia-se que tal questão envolve os parâmetros da compreensão e números e suas relações, conforme Oliveira e Rocha (2011). Abrange o parâmetro da compreensão, tendo em vista que para a resolução da questão faz-se preciso identificar e entender o padrão que se repete na disposição dos números da sequência; e, também, engloba o parâmetro dos números e suas relações em função de ser necessário, para a resolução, a utilização de relações com base em conhecimentos aritméticos.

Dessa forma, com relação aos registros do aluno A, pode-se observar que na Fase 1 o discente esboçou apenas uma resposta concernente à alternativa da questão, deixando as alternativas b e c em branco - alternativas estas que estão sendo levadas em consideração aqui, assim, não é possível identificar nenhum parâmetro conforme designado no parágrafo precedente. Já na Fase 2, o aluno respondeu corretamente à questão, em que se pode identificar os parâmetros da compreensão e dos números e suas relações, conforme registro exposto.

\subsection{Desempenho 2: Respondeu errado na Fase 1 e respondeu certo na Fase 2}

Neste eixo classificatório se enquadram os alunos que, na Fase 1, responderam errado uma ou mais questões e, na Fase 2 , responderam corretamente essas mesmas questões.

Os casos que se encontram nesse eixo são correspondentes aos alunos A, B, D, E, F e G. O aluno A apresenta esse desempenho nas questões 8, 9a), 9b), 13 e 15; o aluno B nas questões 3 e 4c); o aluno D nas questões 3, 4c), 9b) e 9c); o aluno E nas questões 4c) e 8; o aluno F na questão 15; e, o aluno G na questão 3. Podem-se observar tais informações no Quadro 4. 
Quadro 4 -Casos em que ocorrem o desempenho 2

\begin{tabular}{|c|c|c|c|c|c|c|c|c|c|c|c|c|c|c|}
\hline & Q3 & \multicolumn{3}{|c|}{ Q4 } & \multirow[t]{2}{*}{ Q8 } & \multicolumn{3}{|c|}{ Q9 } & \multicolumn{4}{|c|}{ Q10 } & \multirow[t]{2}{*}{ Q13 } & \multirow[t]{2}{*}{ Q15 } \\
\hline A & & $\mathrm{a}$ & b & c & & a & b & c & a & b & c & d & & \\
\hline Aluno $\mathrm{A}$ & & & & & $\mathrm{X}$ & $\mathrm{X}$ & $\mathrm{X}$ & & & & & & $\mathrm{X}$ & $\mathrm{x}$ \\
\hline Aluno B & $\mathrm{X}$ & & & $\mathrm{X}$ & & & & & & & & & & \\
\hline Aluno D & $\mathrm{X}$ & & & $\mathrm{X}$ & & & $\mathrm{x}$ & $\mathrm{X}$ & & & & & & \\
\hline Aluno $E$ & & & & $\mathrm{X}$ & $\mathrm{X}$ & & & & & & & & & \\
\hline Aluno $F$ & & & & & & & & & & & & & & $\mathrm{X}$ \\
\hline Aluno $\mathrm{G}$ & $\mathrm{x}$ & & & & & & & & & & & & & \\
\hline
\end{tabular}

Fonte: os autores.

Referente ao desempenho 2, foi escolhida a questão 3 com relação ao aluno B, conforme se verifica no Quadro 5.

Quadro 5 - Registros do desempenho 2 do Aluno B com relação às fases 1 e 2

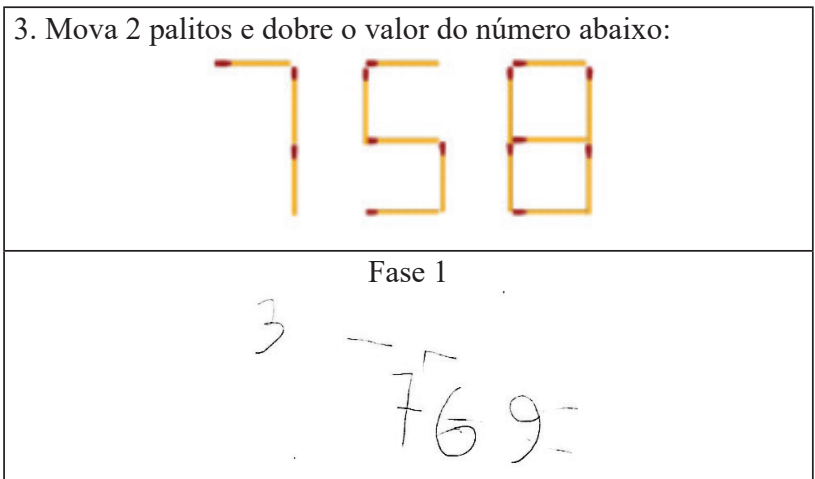

\begin{tabular}{|c|c|c|}
\hline \multicolumn{3}{|l|}{ Fase 2} \\
\hline $3 \quad 15 \mid 6$ & $\begin{array}{l}1400 \\
100\end{array}$ & \\
\hline $\begin{array}{l}\text { Eu Tirei opalito do } 8 \text { e do } 72 \text { assin } \\
\text { Eu formei o Numero } 1516\end{array}$ & $\frac{16}{1516}$ & $\begin{array}{r}11 \\
758 \\
\times 2 \\
1516\end{array}$ \\
\hline
\end{tabular}

Fonte: s autores.

Evidencia-se que esta questão envolve os parâmetros da abstração, compreensão e números e suas relações, em consonância com Oliveira e Rocha (2011). O parâmetro da abstração é elencado, pois para a resolução da questão se subentende a realização de processos mentais, sem o uso de materiais manipuláveis.

Já relativo ao parâmetro da compreensão, faz-se necessário o entendimento da operação de multiplicação para prosseguir com a movimentação dos palitos e encontrar a solução. E quanto ao parâmetro dos números e suas relações, em função do conhecimento atrelado à realização de uma operação aritmética, no caso, o dobro de um número.

Dessa forma, com relação aos registros do aluno B, temse que na Fase 1 o aluno não conseguiu interpretar a questão e, por consequência, não a desenvolveu corretamente, assim, em função dafalta de interpretação, não abrange os parâmetros elencados. Na fase 2, o aluno apresenta os três parâmetros abstração, compreensão, números e suas relações; bem como uma tentativa de argumentação.

\subsection{Desempenho 3: Respondeu certo na Fase 1 e respondeu com registro de resolução e/ou com melhoramento do registro de resolução na Fase 2}

Neste eixo classificatório se enquadram os alunos que, na Fase 1, responderam certo uma ou mais questões e, na Fase 2 , além de responderem corretamente, apresentaram registros de resoluções e/ou algum melhoramento na disposição das respostas em relação a essas mesmas questões. Enfatiza-se que as condições de aplicação dos testes nas duas fases foram as mesmas.

Os casos que se encontram nesse eixo são correspondentes aos alunos A, B, E, F e G. No que se refere ao aluno A, este apresenta o desempenho nas questões 3 e $4 a$ ). O aluno B manifesta nas questões 4a), 4b) e 13. O aluno E, acusa tal desempenho na questão 3. A respeito do aluno $\mathrm{F}$, nota-se na questão 10d). Já o aluno G, acusa esse desempenho na questão 9a). Podem-se observar tais informações no Quadro 6.

Quadro 6 - Casos em que ocorrem o desempenho 3

\begin{tabular}{|c|c|c|c|c|c|c|c|c|c|c|c|c|c|c|}
\hline$Q$ & Q3 & & Q4 & & Q8 & & Q9 & & & & & & Q13 & Q15 \\
\hline A & & a & b & c & & a & b & c & $\mathrm{a}$ & b & c & d & & \\
\hline Aluno A & $\mathrm{X}$ & $\mathrm{X}$ & & & & & & & & & & & & \\
\hline Aluno B & & $\mathrm{X}$ & $\mathrm{X}$ & & & & & & & & & & $\mathrm{X}$ & \\
\hline Aluno E & $\mathrm{X}$ & & & & & & & & & & & & & \\
\hline Aluno $\mathrm{F}$ & & & & & & & & & & & & $\mathrm{X}$ & & \\
\hline Aluno $\mathrm{G}$ & & & & & & $\mathrm{X}$ & & & & & & & & \\
\hline
\end{tabular}

Fonte: os autores.

Levando em consideração o desempenho 3, a questão 4, alternativas a e b, foi escolhida, referente ao aluno B, conforme apresentado no Quadro 7.

Quadro 7 - Registros do desempenho 3 do Aluno B com relação às fases 1 e 2

4. Observe a sequência e escreva o próximo elemento da série: a) $1,3,5,7$,

b) $2,7,12,17,22,27$,

c) $0,1,4,9,16,25,36$,

Fase 1

4

$1,3,5,7,9$

$432,7,12,17,22,27,32$

Fase 2

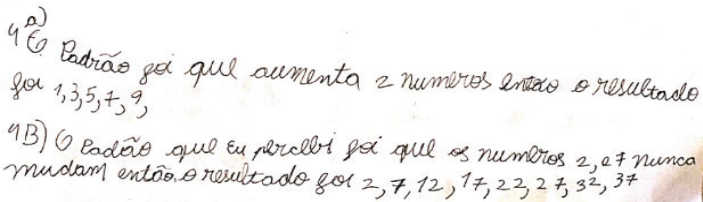

Fonte: Os autores.

Nesse sentido, frisa-se que essa questão envolve os parâmetros da compreensão e números e suas relações, em acordo com Oliveira e Rocha (2011). O parâmetro da compreensão é evidenciado, tendo em vista que para a resolução da questão se faz preciso identificar e entender o 
padrão que se repete na disposição dos números da sequência; e também engloba o parâmetro dos números e suas relações em função de ser necessário, para a resolução, a utilização de relações com base em conhecimentos aritméticos.

Para tanto, com relação aos registros do aluno B, temse que na Fase 1 o aluno apresentou os parâmetros da compreensão e números e suas relações. Por sua vez, na Fase 2, para além desses parâmetros, o aluno também evidenciou os parâmetros da argumentação com base em critérios e em princípios logicamente validados, pois por si só apresentou justificativas para a resolução da questão; e da expressão de ideias, de forma lógica e organizada, tendo em vista que o aluno expôs uma estrutura para a resolução apresentada, explicitando-a.

\subsection{Desempenho 4: Desempenhos mantidos na transição da Fase 1 para a Fase 2}

Neste eixo classificatório se enquadram os alunos que, tanto na Fase 1 quanto na Fase 2, apresentaram desempenho equivalente com relação aos processos de resolução das questões. Nesse caso, salienta-se que nesse eixo estão presentes alunos que responderam corretamente nas duas fases - indicado por X, bem como aqueles que não responderam ou responderam incorretamente ambas as fases - indicado por Y; este também pode ser verificado no caso em que os alunos responderam errado na Fase 1 e não responderam na Fase 2.

Os casos que se encontram nesse eixo são correspondentes aos alunos A, B, C, D, E, F e G. No que se refere ao aluno A, este apresenta o desempenho na questão 10a), 10b), 10c) e 10d); o aluno B nas questões 8, 9a), 9b), 9c), 10a), 10b), 10c), 10d) e 15; o aluno $C$ nas questões 4a), 4b), 8, 9a), 9b), $10 \mathrm{a}), 10 \mathrm{~b}), 10 \mathrm{c}), 10 \mathrm{~d}), 13 \mathrm{e} 15$; o aluno D nas questões $4 \mathrm{a}$ ), 4b), 8, 9a), 10a), 10b), 10c), 10d), 13 e 15; o aluno E nas questões 4a), 4b), 9a), 9b), 9c), 10d), 13 e 15; o aluno $F$ nas questões 4a), 4b), 8, 9a), 9b), 10a), 10b), 10c) e 13; o aluno G nas questões 4a), 4b), 4c), 8, 9b) e 15. Podem-se observar tais informações no Quadro 8.

Quadro 8 - Casos em que ocorre o desempenho 4

\begin{tabular}{|c|c|c|c|c|c|c|c|c|c|c|c|c|c|c|}
\hline Q & Q3 & & Q4 & & Q8 & & Q9 & & & Q & & & Q13 & Q15 \\
\hline A & & a & b & c & & a & b & c & a & b & c & d & & \\
\hline Aluno $\mathrm{A}$ & & & & & & & & & $\mathrm{x}$ & $\mathrm{x}$ & $\mathrm{x}$ & $\mathrm{x}$ & & \\
\hline Aluno B & & & & & $\mathrm{Y}$ & $\mathrm{x}$ & $\mathrm{x}$ & $\mathrm{x}$ & $\mathrm{x}$ & $\mathrm{x}$ & $\mathrm{x}$ & $\mathrm{x}$ & & $\mathrm{Y}$ \\
\hline Aluno C & & $\mathrm{x}$ & $\mathrm{x}$ & & $\mathrm{Y}$ & $\mathrm{Y}$ & $\mathrm{Y}$ & & $\mathrm{x}$ & $\mathrm{x}$ & $\mathrm{x}$ & $\mathrm{x}$ & $\mathrm{Y}$ & $\mathrm{Y}$ \\
\hline Aluno D & & $\mathrm{x}$ & $\mathrm{x}$ & & $\mathrm{Y}$ & $\mathrm{Y}$ & & & $\mathrm{x}$ & $\mathrm{x}$ & $\mathrm{x}$ & $\mathrm{x}$ & $\mathrm{Y}$ & $\mathrm{Y}$ \\
\hline Aluno $E$ & & $\mathrm{x}$ & $\mathrm{x}$ & & & $\mathrm{Y}$ & $\mathrm{Y}$ & $\mathrm{Y}$ & & & & $\mathrm{Y}$ & $\mathrm{Y}$ & $\mathrm{Y}$ \\
\hline Aluno $F$ & & $\mathrm{x}$ & $\mathrm{x}$ & & Y & $\mathrm{x}$ & $\mathrm{x}$ & & $\mathrm{x}$ & $\mathrm{x}$ & $\mathrm{x}$ & & $\mathrm{x}$ & \\
\hline Aluno $\mathrm{G}$ & & $\mathrm{x}$ & $\mathrm{x}$ & $\mathrm{x}$ & $\mathrm{Y}$ & & $\mathrm{Y}$ & & & & & & & $\mathrm{Y}$ \\
\hline
\end{tabular}

Fonte: os autores.

Primeiramente, para exemplificar o desempenho 4, foi escolhida a questão 13 , concernente ao aluno $\mathrm{F}$, conforme se verifica no Quadro 9.
Quadro 9 - Registros do desempenho 4 do Aluno F com relação às fases 1 e 2 - caso 1

13. Observe os grupos de palavras:

I. Seca, peca, saco, naco, taco;

II. Carro, barro, morte, sorte, porte;

III. Pelo, zelo, mente, pente, dente;

IV. Pote, mote, porte, toco, reco;

V. Cama, fama, rama, manda, anda.

Para responder as questões, considere as colocações abaixo:

- As primeiras duas palavras de cada linha rimam;

- As três últimas palavras de cada linha rimam;

- Cada palavra de uma linha começa com uma letra diferente;

- Cada linha contém palavras com o mesmo número de letras.

b) Que linha satisfaz todas as condições dadas?

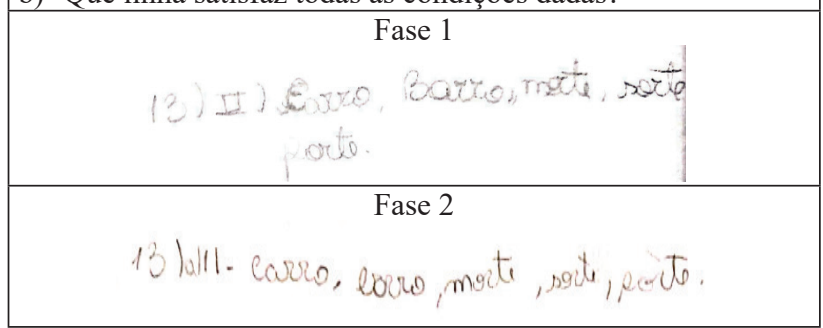

Fonte: os autores.

Os parâmetros evidenciados quanto a essa questão são os da compreensão e expressão de ideias de forma lógica e organizada, segundo Oliveira e Rocha (2011). Tais parâmetros se justificam pelo fato de que se faz necessário a identificação de características que satisfazem as condições apresentadas no enunciado da questão, e a estruturação de uma linha de raciocínio para o processo de resolução da questão com base em eliminação das alternativas que não correspondem a todas as condições impostas, respectivamente.

Nesse sentido, com relação aos registros do aluno $F$, pode-se observar que tanto na Fase 1 quanto na Fase 2, o aluno atingiu o parâmetro da compreensão e da expressão de ideias de forma lógica e organizada, visto que a respondeu corretamente. Nesse caso, o desempenho é evidenciado como respondeu corretamente na Fase 1 e na Fase 2.

Ainda, para exemplificar o desempenho 4, foi escolhida a questão 8 como segundo exemplo, concernente ao aluno $\mathrm{C}$, conforme consta no Quadro 10 a seguir.

Quadro 10 - Registros do desempenho 4 do Aluno C com relação às fases 1 e 2 - caso 2

8. Ana, Beatriz, Célia e Dora apostaram uma corrida.

Ana disse: Célia ganhou, Beatriz chegou em $2^{\circ}$ lugar;

Beatriz disse: Célia chegou em $2^{\circ}$ lugar e Dora, em $3^{\circ}$;

Célia disse: Dora foi a última; Ana, a $2^{\mathrm{a}}$;

Cada uma das meninas disse uma verdade e uma mentira.

Qual a colocação de cada menina?

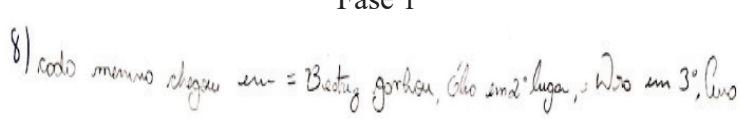

Fase 2

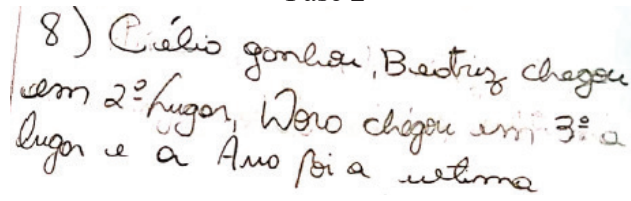

Fonte: Os autores. 
Os parâmetros evidenciados quanto a essa questão são designados pela compreensão e expressão de ideias, de forma lógica e organizada, conforme Oliveira e Rocha (2011). Os parâmetros se justificam, tendo em vista que se faz necessário uma interpretação e tentativa de ordenação com relação ao enunciado da questão.

Dessa forma, na Fase 1 se tem que o aluno respondeu incorretamente, não abrangendo nenhum dos parâmetros, bem como na Fase 2. Nesse caso, o desempenho é evidenciado como não respondeu corretamente na Fase 1 e na Fase 2.

Assim sendo, ao se comparar a Fase 1 e a Fase 2 com relação aos desempenhos apresentados em cada eixo classificatório, observa-se que na Fase 2 os alunos desenvolveram pelo menos os mesmos parâmetros que na Fase 1, além de em alguns casos terem desenvolvido outros a mais; sendo assim, verifica-se que os participantes mantiveram a performance nas questões ou ainda tiveram um progresso com relação às fases.

Destarte, ao analisar os desempenhos apresentados neste escrito, observa-se que houve melhorias nas respostas dos alunos, mesmo que, em algumas vezes, de maneira sutil, conforme apresentado nos exemplos dos desempenhos 1, 2 e 3, elencados anteriormente. Entende-se que as atividades propostas no decorrer das aulas do projeto propiciaram estas melhorias, tendo que em vista que estas envolviam os parâmetros de raciocínio lógico apresentados por Oliveira e Rocha (2011). Para exemplificar essa afirmação se apresenta, a seguir, algumas atividades desenvolvidas no decorrer do projeto e os parâmetros a essas relacionados.

Figura 1 - Uma das atividades desenvolvidas no projeto de robótica

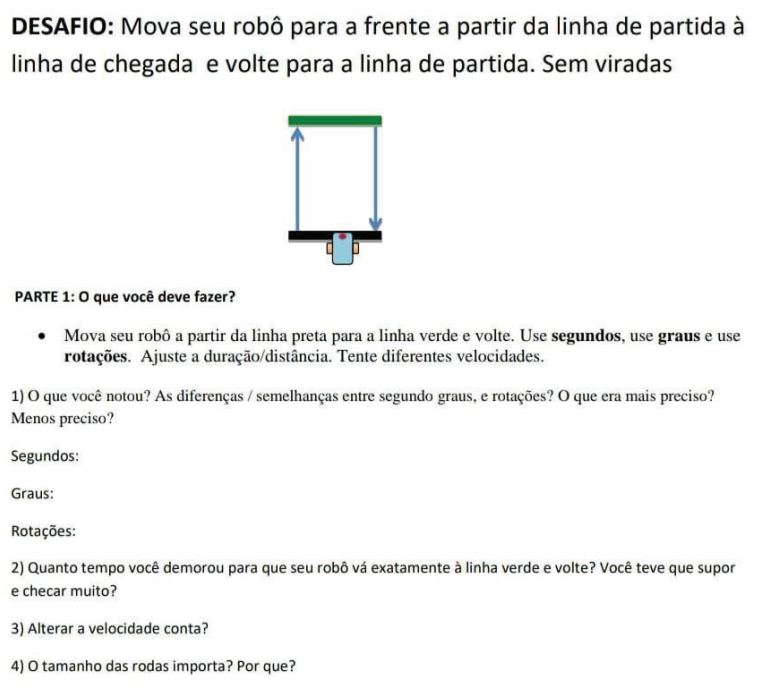

Fonte: Adaptado de Seshan e Seshan (2020).

Nessa atividade é possível identificar os parâmetros compreensão, argumentação e números e suas relações, pois para desenvolver essa atividade foi preciso que os alunos interpretassem o que deveria ser feito para que o robô executasse a ação solicitada, bem como fizessem explicações sobre as relações entre as unidades de medida (segundos, graus e rotações), comparando-as para verificar a precisão de cada uma acerca da distância a ser percorrida. Ao realizar essas ações, entende-se que o parâmetro números e suas relações está envolvido nos processos de cálculo para a realização de conversões entre as unidades de medida.

Figura 2 - Uma das atividades desenvolvidas no projeto de robótica

DESAFIO 1 : Seu robô é um jogador de baseball. Programe o robô para dar a volta no campo de baseball em forma de diamante, cujos lados medem 10 polegadas:

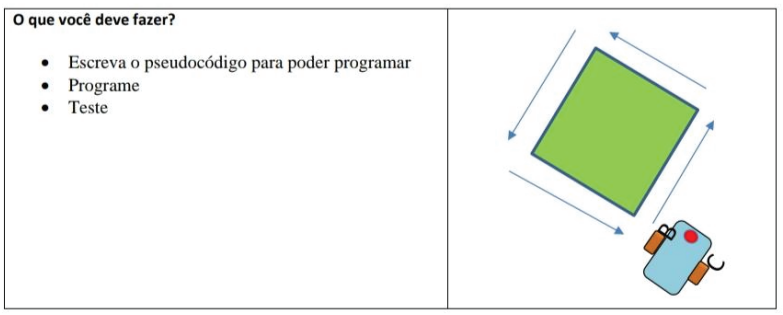

Você pode escrever este pseudocódigo ou em bloco de comentários no EV3.

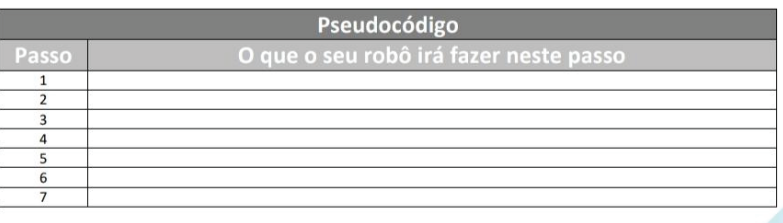

Use o pseudocódigo acima para completar este desafio.

Fonte: Adaptado de Seshan e Seshan (2020).

Nessa atividade é possível perceber os parâmetros abstração, compreensão e expressão de ideias, de forma lógica e organizada, pois para desenvolver essa atividade, os alunos precisavam, inicialmente, interpretar o que foi solicitado para desenvolver o pseudocódigo; ao desenvolver esse pseudocódigo ${ }^{10}$ ele teria que imaginar o percurso/caminho a ser percorrido pelo robô para descrever os possíveis comandos a serem realizados, e esta ação exige uma organização do pensamento de forma lógica e estruturada.

Nesse sentido, embora o foco deste trabalho não sejam as atividades desenvolvidas ao longo do projeto de robótica, ressalta-se que estas contribuíram para as melhorias destacadas anteriormente, no que diz respeito aos desempenhos da Fase 1 para a Fase 2. Assim sendo, pode-se afirmar que as melhorias apresentadas na Fase 2 ocorreram em decorrência das atividades realizadas no decorrer do projeto, possibilitando o desenvolvimento do raciocínio lógico por parte dos alunos.

\section{Conclusão}

Este escrito teve como objetivo verificar desempenhos ocorridos nos processos de resolução de questões envolvendo raciocínio lógico, com a participação de alunos do $7^{\circ}$ ano do Ensino Fundamental, no que diz respeito às fases iniciais e finais de um projeto de robótica. Dessa forma, com o intuito

10 Pseudocódigo: linguagem de computador textual simplificada, que não segue especificamente a sintaxe de nenhuma das linguagens de computador existentes (GONZALES, 2016). 
de atender ao objetivo proposto, foram realizadas análises com relação aos registros dos alunos, as quais possibilitaram a identificação de quatro tipos de desempenhos, denotados por eixos classificatórios.

Nos desempenhos 1, 2 e 3 foi possível observar que os alunos manifestaram melhorias com relação ao desenvolvimento do raciocínio lógico, pois se notou que na Fase 2 foram identificados parâmetros que não estavam presentes na Fase 1. Essa ideia é corroborada na discussão apresentada na análise dos dados, realizada com base nos pressupostos elencados no referencial teórico deste trabalho.

Em virtude disso, pode-se inferir que as atividades desenvolvidas ao longo do projeto de robótica propiciaram o desenvolvimento do raciocínio lógico por parte dos alunos, o que foi verificado em registros da Fase 2, e isso contribui para possíveis mobilizações de conhecimentos matemáticos dos educandos que apresentavam dificuldades na disciplina de Matemática.

Destaca-se que, além dos desempenhos que foram elencados no trabalho, em alguns casos houve regressos em relação aos registros de resolução das questões, tendo em vista que na Fase 1 alguns alunos responderam à questão, e na Fase 2 deixaram de responder. Isso pode ser justificado pelo fato de que, na Fase 2, os alunos se dedicaram mais ao desenvolvimento das questões, não meramente chutando possíveis resultados e buscando, várias vezes, por possibilidades de respostas, o que fez com que não conseguissem finalizar todas as questões dentro do tempo disponível, gerando essa ocorrência.

Ademais, evidencia-se que este trabalho está relacionado ao primeiro ano de aplicação do projeto: "Projeto Lapidando Joias: um resgate ao futuro", sendo assim, atenta-se para o fato de que para os próximos anos se tem a necessidade de se intensificar o ensino de Matemática atrelado à robótica, planejando atividades que explorem mais a fundo alguns conteúdos específicos da disciplina, colaborando mais intensamente com a aprendizagem matemática dos alunos participantes. Também como futuras investigações, pretendese investigar os princípios do pensamento computacional que emergem da realização de atividades que envolvem a robótica.

\section{Referências}

BRACKMANN, C.P.; CAETANO, S.V.N.; SILVA, A.R. Pensamento computacional desplugado: ensino e avaliação na educação brasileira primária. Renote, v.17, n.3, p.636-647, 2019. doi: https://doi.org/10.22456/1679-1916.99894.

BRASIL. Secretaria de Educação Fundamental. Parâmetros Curriculares Nacionais: matemática. Brasília: MEC, 1997.

BRASIL. Ministério da Educação. Base Nacional Comum Curricular. Educação Infantil, Ensino Fundamental e Ensino
Médio. Brasília: MEC, 2018.

CIRINO, H.F.F. Lógica Matemática e Lógica Digital. Campinas: Papirus, 1984.

CROVADOR, Á. Física aplicada à robótica. Curitiba: Contentus, 2020.

D'AMBROSIO, B. Como ensinar matemática hoje. Temas e Debates, v.2, n.2, p.15-19, 1989.

DESEMPENHO. In: Michaelis, Dicionário Brasileiro da Língua Portuguesa. Editora Melhoramentos Ltda, 2020. Disponível em: $\quad<$ https://michaelis.uol.com.br/moderno-portugues/busca/ portugues-brasileiro/desempenho>. Acesso em: 18 jul. 2020.

FONSECA, R.V. Desafios geométricos com palitos de fósforos. Belém: UEPA, 2011.

GONZALES, M.R. Codigo alfabetización y pensamiento computacional en educación primaria y secundaria: validación de un instrumento y evaluación de programas. Madrid: UNED, 2016.

MORGADO, A.C.; CESAR, B. Raciocínio Lógico-Quantitativo: teoria, questões resolvidas, questões de concursos, mais de 850 questões. Rio de Janeiro: Elsevier, 2008.

NEGREIROS, T.D.V.; MIRANDA, D.F. Caderno de atividades raciocínio lógico: uma contribuição para a organização do pensamento. 2015. Disponível em: <http://www1. pucminas.br/imagedb/documento/DOC_DSC_NOME_ ARQUI20160317141310.pdf>. Acesso em: $27 \overline{\text { jul }} 20 \overline{2} 1$.

OLIVEIRA, P.A.; ROCHA, A.J.O. Raciocínio lógico, conceitos e estabelecimento de parâmetros para a aprendizagem matemática. 2011. Disponível em: <https://goo.gl/tMiljh>. Acesso em: 18 jul. 2020.

PONTES, E.A.S. et al. Raciocínio lógico matemático no desenvolvimento do intelecto de crianças através das operações de adição e subtração. Diversitas J., v.2, n.3, p.469-476, 2017. doi: https://doi.org/10.17648/diversitas-journal-v2i3.552.

ROSA, P.R.S. Uma introdução à pesquisa qualitativa em ensino de ciências. Campo Grande: Universidade Federal do Mato Grosso do Sul, 2013.

SCOLARI, A.T.; BERNARDI, G.; CORDENONSI, A.Z. O desenvolvimento do raciocínio lógico através de objetos de aprendizagem. Renote, v.5, n.2, p.1-10, 2007. doi: https://doi. org/10.22456/1679-1916.14253.

SESHAN, S.; SESHAN, A. Ev3 Lessons. Pittsburgh, 2020. Disponível em: $<$ https://ev3lessons.com/en/index.html $>$. Acesso em: 18 jul. 2020.

SERATES, J. Raciocínio Lógico: lógico matemático, lógico quantitativo, lógico numérico, lógico analítico, lógico crítico. Brasília: Gráfica e Olímpica, 1997.

SILVA, S. O. A.; LUNA, S.V. Correlação entre o raciocínio lógico e o raciocínio matemático em crianças escolarizadas. Bolema, v.33, n.65, p.1047-1066, 2019. doi: http://dx.doi. org/10.1590/1980-4415v33n65a04.

VAN DE WALLE, J.A. Tecnologia e Matemática Escolar. In: VAN DE WALLE, J. A. (Org.). Matemática no ensino fundamental: formação de professores e aplicação em sala de aula. Tradução: Paulo Henrique Colonese. Longman: Penso, 2009, p. 130-143. 